\title{
DESEMPENHO DA ESTRATIFICAÇÃO EM UM FRAGMENTO DE CERRADO STRICTO SENSU UTILIZANDO INTERPOLADOR GEOESTATÍSTICO
}

\author{
Luiz Henrique Victor Alvarenga ${ }^{1}$, José Márcio de Mello ${ }^{2}$, Isabel Carolina de Lima Guedes ${ }^{3}$, José Roberto Soares Scolforo ${ }^{4}$
}

(recebido: 10 de setembro de 2010; aceito: 31 de agosto de 2012)

\begin{abstract}
RESUMO: Conduziu-se este estudo, com o objetivo de verificar a existência de dependência espacial em um fragmento de cerrado stricto sensu e incorporá-la ao inventário florestal como estratificador e comparar o desempenho da amostragem casual estratificada (ACE) com a amostragem sistemática (AS) além de verificar a precisão da ACE com intensidade amostral reduzida. Foram distribuídas de forma sistemática 157 unidades amostrais, com área de $1000 \mathrm{~m}^{2}$. Em todas as parcelas, foram medidas as circunferências a 1,3 m do solo e altura total de todas as árvores. O volume de cada parcela foi obtido por equações de volume geradas para a fisionomia. Verificou-se que a característica dendrométrica volume se encontra estruturada espacialmente. $\mathrm{O}$ erro de amostragem do inventário obtido para a AS foi de $11,38 \%$ e $6,47 \%$ para ACE. Com a intensidade amostral reduzida em $60 \%$, o erro para ACE foi de $9,93 \%$. Foi verificado que mesmo com uma redução acentuada na intensidade amostral, as estimativas da ACE são mais precisas que as estimativas da AS. Portanto, pode-se dizer que, a estratificação com base na dependência espacial da característica de interesse, torna-se uma ferramenta muito útil na melhora da qualidade dos estimadores do inventário florestal mesmo com redução da intensidade amostral.
\end{abstract}

Palavras-chave: Inventário florestal, estatística espacial, interpolador espacial, krigagem.

\section{PERFORMANCE OF STRATIFICATION IN A BRAZILIAN SAVANNA FRAGMENT BY USING GEOESTATISTICAL INTERPOLATOR}

\begin{abstract}
This study verified the existence of spatial dependence in a Brazilian savanna fragment and incorporated it to the forest inventory by stratifying, compared the accuracy of systematic sampling (SS) with stratified random sampling (SRS) and verified the accuracy of SRS with reduced sampling intensity. A total of 157 sample plots (area of $1000 \mathrm{~m}^{2}$ ) were allocated and distributed systematically in the area. The circumference at $1.3 \mathrm{~m}$ from the soil and the total height of all trees were measured in the plots. The volume of each plot was obtained by volume equations generated for the physiognomy. It was found that the dendrometric characteristic of volume is spatially structured. The inventory error obtained for the SS was $11.38 \%$ and 6,47\% for SRS. With the sampling intensity decreased by $60 \%$, the error for SRS was $9.93 \%$. These results showed that even with a marked reduction in the sampling intensity, the estimates by stratified random sampling (SRS) is more accurate than the estimates of systematic sampling (SS). Therefore, the stratification based on the spatial dependence in the characteristic of interest is a very useful tool in improving the quality of forest inventory estimators even with a reduction in sampling intensity.
\end{abstract}

Key words: Forestry inventory, spatial statistic, spatial interpolator, kriging.

\section{INTRODUÇÃO}

O cerrado ocupa aproximadamente $21 \%$ do território nacional, sendo o segundo maior bioma brasileiro, além de ser considerada a última fronteira agrícola do mundo (BORLAUG 2002). Com a ocupação do homem e o crescente desenvolvimento, a destruição dos ecossistemas que constituem o cerrado se agrava cada vez mais. Estima-se que $55 \%$ da área original do cerrado foram desmatadas ou modificadas pela ação antrópica (KLINK; MACHADO, 2005).
Para garantir a sustentabilidade no processo de exploração dos recursos naturais necessários ao desenvolvimento, adotam-se práticas de manejo sustentado. Ele propicia uma série de bens, para os quais não há mercado perfeito e que são de difícil mensuração em termos monetários. O manejo sustentável é aquele que favorece uma produção contínua do recurso explorado, sem a deterioração dos demais recursos e benefícios envolvidos (SCOLFORO, 1998). Para a efetivação com qualidade de um plano de manejo, é de fundamental

\footnotetext{
${ }^{1}$ Engenheiro Florestal, Mestre em Engenharia Florestal - Plantar - Av. Raja Gabaglia, 1380, Gutierrez - $30440-452$ - Belo Horizonte, MG, Brasil - lhvalvarenga@gmail.com

${ }^{2}$ Engenheiro Florestal, Professor Doutor em Recursos Florestais - Universidade Federal de Lavras/UFLA - Departamento de Ciências Florestais - Cx. P. 3037 - 37200-000 - Lavras, MG - josemarcio@dcf.ufla.br

${ }^{3}$ Engenheira Florestal, Doutora em Engenharia Florestal - Plantar - Av. Raja Gabaglia, 1380, Gutierrez - $30440-452$ - Belo Horizonte, MG, Brasil - isabelcarolinadelima@yahoo.com.br

${ }^{4}$ Engenheiro Florestal, Professor Doutor em Engenharia Florestal - Universidade Federal de Lavras - Departamento de Ciências Florestais - Cx. P. 3037 - 37200-000 - Lavras, MG - jscolforo@dcf.ufla.br
} 
importância conhecer o potencial produtivo. Esse conhecimento se fundamenta no sistema de amostragem adotado na área objeto de manejo.

Segundo Assis et al. (2009), para promover a otimização do uso dos recursos florestais é fundamental conhecê-los, quantificá-los e monitorá-los de maneira adequada. Isso só é possível utilizando técnicas de amostragem que permitem a obtenção de informações confiáveis com custos oportunos, onde o monitoramento dos recursos é realizado por uma amostra representativa da população. O inventário florestal utiliza os conceitos da teoria da amostragem para a estimativa de características quantitativas e, ou qualitativas da floresta (MELLO et al., 2009).

Segundo Péllico Netto e Brena (1997), amostragem casual simples é considerada um procedimento de amostragem de aleatoriedade irrestrita. $\mathrm{O}$ fundamento para a aplicação desse procedimento é que a floresta seja homogênea na característica de interesse. Quando o atributo de interesse apresenta alto grau de variabilidade, uma alternativa para a melhoria da precisão é a estratificação.

A amostragem casual estratificada consiste na divisão da área em subpopulações mais homogêneas na característica avaliada. A estratificação pode ser baseada em informações cadastrais, imagens de satélite ou ainda em informações da própria característica, por meio de interpoladores espaciais. O fato de se obter estratos homogêneos na variável estudada contribui para o aumento da precisão do levantamento (ASSIS et al., 2009; COCHRAN, 1963; KANEGAE JÚNIOR et al., 2006; MCROBERTS et al., 2002).

Os estimadores usados no inventário florestal são os da estatística clássica e devem respeitar o princípio de que as amostras são independentes entre si e seguem uma distribuição normal. Contudo, diversos trabalhos desenvolvidos na área florestal verificaram presença de estrutura de continuidade espacial nas características dendrométricas avaliadas (ASSIS et al., 2009; KANEGAE JÚNIOR et al., 2007; MELLO et al., 2005a, 2005b, 2006, 2009).

Assim, uma análise variográfica se faz necessária antes do processamento do inventário florestal, pois, se houver dependência espacial entre as amostras, esta deve ser considerada nas análises a fim de se obter estimativas mais precisas e confiáveis (ASSIS et al., 2009).

Diante do exposto, os objetivos deste estudo foram: i) verificar a existência de dependência espacial da variável volume em um fragmento de Cerrado Stricto Sensu; ii) realizar a estratificação com base no interpolador geoestatístico; iii) comparar a precisão da amostragem sistemática, usando os estimadores da amostragem casual simples, com a amostragem casual estratificada gerada pelo interpolador geoestatístico; iii) avaliar o impacto da redução da intensidade amostral sobre a precisão do inventário.

\section{METODOLOGIA}

\subsection{Caracterização da área e coleta dos dados}

O fragmento está situado no município de São Romão, MG, com área de 7.706,59 hectares, cuja fitofisionomia é do tipo Cerrado Sensu Stricto. A altitude média do municípioé de 425,5 metros e o clima segundo a classificação de Köppen é do tipo Aw, caracterizado por clima tropical com estação seca no inverno. A temperatura média do mês mais frioé superior a $18^{\circ} \mathrm{C}$ (NIMER, 1979). O tipo de solo predominante é o Neossolo flúvico (SCOLFORO et al., 2008).

Foram distribuídas de forma sistemática 157 unidades amostrais georeferenciadas de $1000 \mathrm{~m}^{2}$ cada, com distância de 700 metros entre si. Todas as parcelas foram medidas as circunferências a 1,30 m do solo (CAP) de todas as árvores com $\mathrm{CAP} \geq 15,7 \mathrm{~cm}(\mathrm{DAP}=5 \mathrm{~cm})$ e suas respectivas alturas. $\mathrm{O}$ volume foi obtido por equação gerada para a fitofisionomia, disponíveis em Rufini et al. (2010).

\subsection{Estudo variográfico e interpolação espacial}

Com o intuito de entender o comportamento geral dos dados, foi realizada a análise exploratória. Foram determinadas as medidas de posição, dispersão, avaliação da normalidade pelo teste de Shapiro-Wilk com um nível de significância $\alpha=5 \%$ (FERREIRA, 2005), análise de tendência da disposição das amostras em relação à latitude e à longitude e identificação de valores discrepantes.

O estudo variográfico foi realizado por meio da confecção do semivariograma experimental, que permite verificar a estrutura da dependência espacial do volume $\left(\mathrm{m}^{3} / \mathrm{ha}\right)$. O estimador da semivariância é igual à média aritmética das diferenças ao quadrado entre pares de valores experimentais, em todos os pontos separados pela distância $h$ (JOURNEL; HUIJBREGTS, 1978).

$$
\hat{y}(h)=\frac{1}{2 N(h)} \sum_{i=1}^{N(h)}\left[Z\left(x_{i}\right)-Z\left(x_{i}+h\right)\right]^{2}
$$


Emque:

$\hat{y}(h)$ é a semivariância estimada entre pares de pontos, na mesma distância $h$;

$N(h)$ é o número de pares de pontos separados por um vetor $h$;

$Z\left(x_{i}\right)$ é o valor da variável regionalizada no ponto $x$; $Z\left(x_{i}+h\right)$ é o valor no ponto $x+h$.

Ao semivariograma experimental foram ajustados: modelos esférico e exponencial utilizando: métodos dos quadrados mínimos ponderados e quadrados mínimos ordinários. Após os ajustes dos modelos, faz-se necessária a seleção do melhor modelo ajustado ao semivariograma experimental. Segundo Vendrusculo (2003), o processo de seleção é crucial para a correta interpretação de fenômenos com dependência espacial, além disso, essa escolha influencia diretamente na confiabilidade dos resultados oriundos do interpolador geoestatístico.

O processo de seleção na modelagem geoestatística inicia-se com a validação cruzada. Posteriormente, podese avaliar o grau de dependência espacial (DE) que é uma relação percentual entre a variação estruturada $\sigma^{2}$ em relação ao patamar $\left(\tau^{2}+\sigma^{2}\right)$, apresentado por Biondi et al. (1994). Burnhan e Anderson (2002) propuseram o uso do critério de informação AIC (Akaike's Information Criterion) apresentado por Akaike (1983).

Após a obtenção do modelo espacial, foi realizada a interpolação por meio da krigagem, um interpolador geoestatístico, desenvolvido por Matheron (1963) que estima os valores de pontos não amostrados com base nos pontos amostrados, levando em consideração a estrutura de dependência espacial. A krigagem da área de estudo foi realizada considerando cinco classes de produtividade.

\subsection{Processamento do inventário}

O processamento do inventário foi realizado de duas maneiras: i) considerando todas as unidades amostrais dentro de uma população não estratificada, utilizando os estimadores da Amostragem Casual Simples (ACS); ii) considerando a estratificação da população pelo interpolador geoestatístico, onde o processamento foi realizado com base nos estimadores da Amostragem Casual Estratificada (ACE).

A fim de verificar o ganho, não somente em precisão, mas também em custo, efetuou-se o processamento de ACE com uma redução de aproximadamente $60 \%$ na intensidade amostral em cada estrato. Para verificar a estabilidade dos estimadores com essa redução, foram efetuadas 1000 simulações em cada estrato, retirando-se aleatoriamente $40 \%$ das parcelas ali existentes. Posteriormente, para cada estrato, obtiveram-se as estimativas médias das simulações para as estatísticas do inventário.

A precisão dos procedimentos foi avaliada pelo erro de amostragem em porcentagem. Com esta comparação, objetivou-se avaliar a qualidade e o potencial do uso do interpolador geoestatístico para obtenção de estratos. A formulação detalhada dos procedimentos de amostragem utilizados pode ser encontrada em Cochran (1963), Péllico Netto e Brena (1997) e Scolforo e Mello (2006).

\section{RESULTADOS E DISCUSSÃO}

\subsection{Análise variográfica}

Pela análise exploratória dos dados de volume $\left(\mathrm{m}^{3} / \mathrm{ha}\right)$ foi observado um valor médio de $33,18 \mathrm{~m}^{3} / \mathrm{ha}$, desvio padrão de $23,97 \mathrm{~m}^{3} /$ ha e coeficiente de variação de $72,2 \%$, mostrando alto grau de variabilidade, o que justifica a realização da estratificação. Pelo teste de Shapiro-Wilk foi possível observar que, a 5\% de probabilidade os valores de volume $\left(\mathrm{m}^{3} / \mathrm{ha}\right)$ não apresentaram distribuição aproximadamente normal. Essa informação é importante, porque permite adotar métodos de ajuste que demandam o conhecimento a priori da distribuição dos dados, como é o caso do método da Máxima Verossimilhança.

Não foram observadas tendências em relação aos sentidos norte-sul e leste-oeste e presença de dados discrepantes. Os valores discrepantes podem influenciar o resultado do semivariograma experimental, principalmente na sua porção inicial (CRESSIE, 1993; RIBEIRO JÚNIOR, 1995), enquanto que a presença de tendência pode mascarar a estrutura de dependência espacial ou ainda apresentar uma falsa estrutura de dependência.

Na Figura 1, mostra-se o semivariograma experimental e os modelos a eles ajustados pelos métodos dos mínimos quadrados ponderados e ordinários. Foi possível detectar que há uma estrutura correlacionada espacialmente da característica volume, independente do modelo e método de ajuste avaliado. Verificou-se, pela Figura 1, que o modelo exponencial foi aquele que teve o menor efeito pepita, que é a parte do erro não explicada pelo modelo. Isso implica em estimativas mais precisas no processo da krigagem.

Na Tabela 1, mostram-se os parâmetros efeito pepita $\left(\tau^{2}\right)$, que indicam a variação aleatória; patamar $\left(\sigma^{2}\right)$, variação explicada pela componente espacial e alcance $(\phi)$, ponto a partir do qual os dados se comportam de forma independente; grau de dependência espacial (DE) e o critério de informação de Akaike (AIC) para os métodos de ajuste e modelos utilizados. Essas informações auxiliam na definição do melhor modelo e do melhor método de ajuste.

Cerne, Lavras, v. 18, n. 4, p. 675-681, out./dez. 2012 

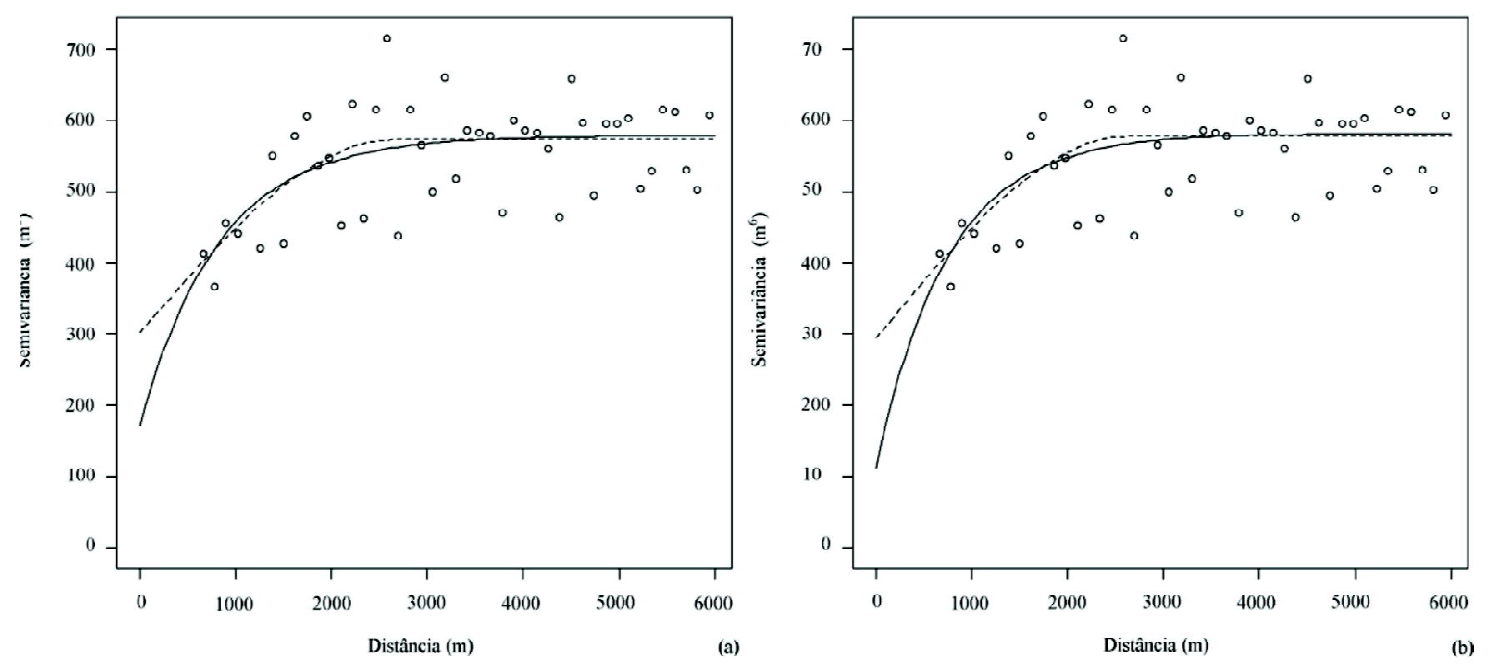

Figura 1 - Ajuste dos modelos exponencial (linha cheia) e esférico (linha tracejada) pelo método dos mínimos quadrados ponderados (a) e mínimos quadrados ordinários (b).

Figure 1 - Adjustment of the exponential model (solid line) and spherical model (dashed line) by the methods WLS(a) and OLS (b).

Tabela 1 - Parâmetros dos modelos, grau de dependência espacial (DE) e critério de informação de Akaike (AIC).

Table 1 - Model parameters, degree of spatial dependence (SD) and Akaike Information Criterion (AIC)

\begin{tabular}{ccccccc}
\hline Método de Ajuste & Modelo & $\tau^{2}$ & $\sigma^{2}$ & $\phi^{*}$ & DE & AIC \\
\hline \multirow{2}{*}{ OLS } & Exponencial & 112,48 & 468,60 & 751,60 & 80,64 & 1431,48 \\
& Esférico & 293,97 & 282,79 & 2612,50 & 49,03 & 1432,85 \\
\hline \multirow{2}{*}{ WLS } & Exponencial & 171,46 & 406,57 & 827,84 & 70,33 & 1431,72 \\
& Esférico & 302,15 & 269,94 & 2630,38 & 47,18 & 1432,76 \\
\hline
\end{tabular}

* Para o modelo exponencial, trata-se do alcance teórico para ambos os métodos de ajuste.

Segundo Burnhan e Anderson (2002), os modelos são considerados semelhantes entre si pois a diferença entre os valores de AIC é menor que 2. Contudo, foi selecionado o modelo exponencial ajustado pelo método dos Quadrados Mínimos Ordinários (OLS) por apresentar o menor valor de AIC (MCBRATNEY; WEBSTER, 1986), além de apresentar o maior grau de dependência espacial (DE). Esse fato, pode ser observado na Figura 1, onde se nota claramente que o modelo com o ajuste OLS teve o menor efeito pepita.

$\mathrm{O}$ efeito pepita sendo diferente de zero indica que nem toda a variação pode ser explicada pela componente espacial. Isso ocorre por falta de amostragem nas distâncias menores que a menor distância entre as parcelas. No presente trabalho, a menor distância média entre as parcelas foi de 700 metros.

No modelo exponencial, o alcance fornecido é o alcance teórico, sendo que o alcance prático deve ser multiplicado por três. Nesse caso, o alcance teórico foi de 751,60 metros. Na situação do presente estudo, levantamentos com amostras distantes entre si superiores a 2.254,80 metros podem ser tratados pela estatística clássica. Para os casos em que a distância entre as amostras for menor que o valor do alcance, a estrutura de continuidade espacial deve ser considerada nas análises.

Segundo Biondi et al. (1994), quando a dependência espacial $(\mathrm{DE})>75 \%$, o grau de dependência espacial é considerado alto; $35 \leq \mathrm{DE} \leq 75 \%$, médio grau de dependência espacial e $<35 \%$, fraca estrutura de dependência espacial. Todos os modelos pelos dois métodos de ajuste evidenciaram uma estrutura de continuidade espacial de média a forte. No presente estudo, o grau de dependência espacial para volume foi de 80,64\%. Dessa forma, a estrutura de correlação entre as parcelas nas análises estatísticas, melhora a qualidade da precisão nas estimativas.

\section{Cerne, Lavras, v. 18, n. 4, p. 675-681, out./dez. 2012}


Nos projetos com grau de dependência espacial de médio a forte, é possível gerar mapas que correspondem a uma pós-estratificação com base no volume, sem tendência e não enviesados, isto é, mais precisos no detalhamento espacial da variável dendrométrica de interesse (ASSIS et al., 2009). Na Figura 2, apresenta-se o mapa da pósestratificação obtido para o fragmento.

\subsection{Processamento do inventário}

Utilizando-se as 157 parcelas do inventário, o precessamento foi realizado de 2 formas: pela amostragem

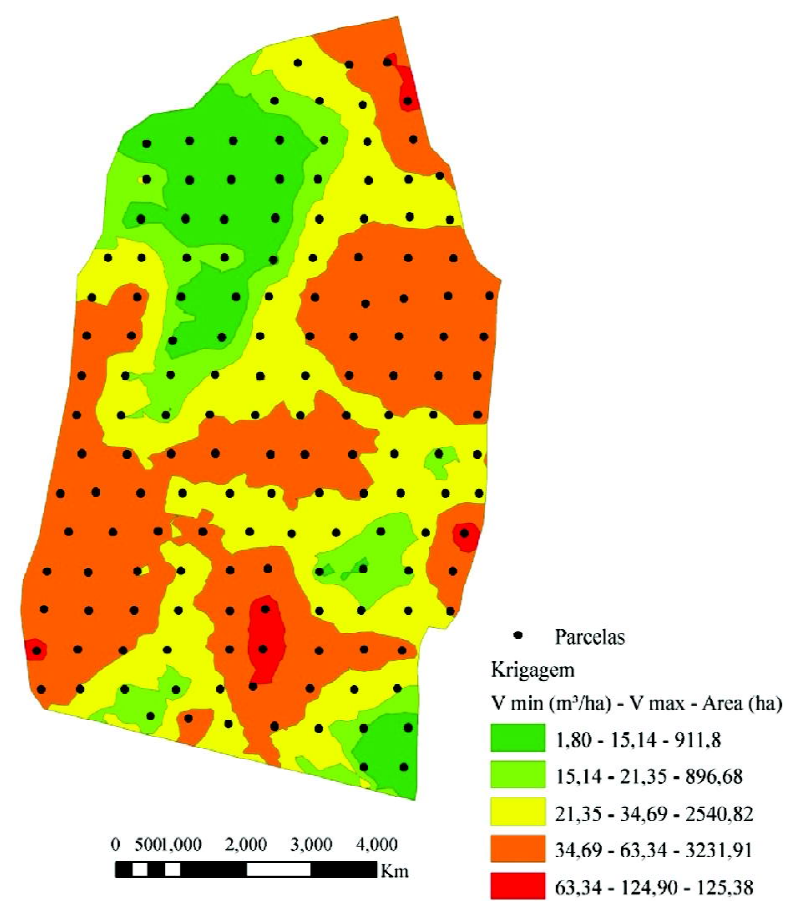

Figura 2 - Resultado da krigagem obtida na área de estudo.

Figure 2-Results of kriging obtained of the studied area. sistemática (AS) e pela amostragem casual estratificada (ACE) gerada pela interpolação geoestatística. Posteriormente, a amostragem casual estratificada (ACE) foi realizada utilizando 65 parcelas das 157 totais amostradas. A escolha das parcelas por estrato levou em consideração a distribuição das mesmas dentro do estrato gerado pela krigagem e a quantidade de parcelas por estrato. Sendo assim, foram aleatorizadas $8,8,21,26$ e 2 parcelas para os estratos das classes I, II, III, IV e V, respectivamente, sendo estas ordenadas da menor para a maior classe de produtividade.

Os resultados do processamento do inventário, considerando os dois procedimentos de amostragem estão apresentados na Tabela 2. O erro de amostragem do inventário foi de $11,38 \%$ para a AS, de $6,47 \%$ para a ACE e 9,93\% para ACE com redução da intensidade amostral em $60 \%$ (65 parcelas). Os intervalos de confiança apresentados na Tabela 2 foram semelhantes. Isso evidencia que a técnica de estratificação (mesmo com a redução da intensidade amostral), reduz o erro de amostragem do inventário, gera estimativa do parâmetro próxima daquele que envolve um número elevado de parcelas.

Resultados satisfatórios foram encontrados também por Mello e Scolforo (2000), quando, utilizando-se do procedimento de estratificação, observou uma redução no erro de amostragem na ordem de $29,42 \%$, quando comparada com a AS.

Silva (2009) estratificou fragmentos de cerrado no estado de Minas Gerais com base em imagens de satélite (préestratificação) e constatou que para reduções de 20 e $40 \%$ na intensidade amostral, o erro de amostragem do inventário (\%) apresentava pequeno aumento, porém, esse aumento não ultrapassou o limite imposto pela legislação vigente.

Com base nos resultados obtidos, nota-se que a estratificação é uma ferramenta valiosa não somente para o aumento da precisão, mas também para a redução de tempo de execução do inventário, quando se admite um erro máximo pré-estabelecido e, consequentemente, de custo.

Tabela 2 - Amostragem sistemática (AS); Amostragem casual estratificada (ACE) e Amostragem casual estratificada com redução de 60\% no número de parcelas (ACE (60\%)).

Table 2 - Systematic sampling (SS); Stratified random sampling (ACE) and Stratified random sampling with a reduction of $60 \%$ in the number of plots (ACE (60\%)).

\begin{tabular}{lccc}
\hline Estimadores & AS & ACE & ACE $(60 \%)$ \\
\hline Média $\left(\mathrm{m}^{3} \cdot \mathrm{ha}^{-1}\right)$ & 33,18 & 32,74 & 32,70 \\
Desvio Padrão $\left(\mathrm{m}^{3} \cdot \mathrm{ha}^{-1}\right)$ & 23,97 & 13,04 & 12,95 \\
Erro do inventário $\left(\mathrm{m}^{3} \cdot \mathrm{ha}^{-1}\right)$ & 3,7755 & 2,1195 & 3,2499 \\
Erro de amostragem $(\%)$ & 11,38 & 6,47 & 9,93 \\
Intervalo de Confiança $\left(\mathrm{m}^{3} \cdot \mathrm{ha}^{-1}\right)$ & $29,40-36,95$ & $30,62-34,86$ & $29,45-35,95$ \\
\hline
\end{tabular}

Cerne, Lavras, v. 18, n. 4, p. 675-681, out./dez. 2012 


\section{CONCLUSÕES}

Com base nos resultados obtidos pode ser concluir que:

Foi detectada a existência de dependência espacial para a variável volume, em fragmento de Cerrado Sensu Stricto. Isso implica que a correlação existente deve ser incorporada em análises estatísticas futuras, visando a uma melhor precisão das estimativas;

Os estimadores da amostragem casual estratificada foram mais precisos que a da amostragem sistemática, indicando que o uso do interpolador geoestatístico para a estratificação da população foi eficiente;

Com a redução da intensidade amostral, o erro de amostragem do processo casual estratificada (ACE) foi menor que o da amostragem sistemática (AS). Isso indica que, sempre que for possível realizar uma estratificação confiável da característica de interesse, a estimativa de volume por meio de ACE é mais precisa quando comparada à estimativa pela $\mathrm{AS}$.

\section{REFERÊNCIAS}

AKAIKE, H. Information measures and model selection. International Statistical Institute, Voorburg, v. 44, p. 277 $291,1983$.

ASSIS, A. L.; MELLO, J. M.; GUEDES, I. C. L.; SCOLFORO, J. R. S.; OLIVEIRA, A. D. Development of a sampling strategy for young stands of Eucalyptus sp. using geoestatistics. Cerne, Lavras, v. 15, n. 2, p. 166173, 2009.

BIONDI, F.; MYERS, D. E.; AVERY, C. C. Geostatistically modelling stem size and increment in an old growth forest. Canadian Journal of Forest Research, Ottawa, v. 24, n. 7, p. 1354-1368, July 1994.

BORLAUG, N. E. Feeding a world of 10 billion people: the miracle ahead. In: BAILEY, R. (Ed.). Global warming and other eco-myths. Roseville: Competitive Enterprise Institute, 2002. p. $29-60$.

BURNHAN, K. P.; ANDERSON, D. R. Model selection and multimodel inference. $2^{\text {nd }}$ ed. New York: Springer, 2002. $350 \mathrm{p}$.

COCHRAN, W. G. Técnicas de amostragem. Rio de Janeiro: USAID, 1963. 555 p.

Cerne, Lavras, v. 18, n. 4, p. 675-681, out./dez. 2012
CRESSIE, A. G. Statistics for spatial data. New York: Wiley, 1993. $900 \mathrm{p}$.

FERREIRA, D. F. Estatística básica. Lavras: UFLA, 2005. $664 \mathrm{p}$.

JOURNEL, A. G.; HUIJBREGTS, C. J. Mining

geostatistics. London: Academic, 1978. 600 p.

KANEGAE JÚNIOR, H.; MELLO, J. M.; SCOLFORO, J. R. S.; OLIVEIRA, A. D. Avaliação da continuidade espacial de características dendrometricas em diferentes idades de povoamentos clonais de Eucalyptus sp. Revista Árvore, Viçosa, v. 31, n. 5, p. 859-866, 2007.

KANEGAE JÚNIOR, H.; SCOLFORO, J. R. S.; MELLO, J. M.; OLIVEIRA, A. D. Avaliação de interpoladores estatísticos e determinísticos como instrumento de estratificação de povoamentos clonais de Eucalyptus sp. Cerne, Lavras, v. 12, n. 2, p. 123-136, abr./jun. 2006.

KLINK, C. A.; MACHADO, R. B. A conservação do cerrado brasileiro. Megadiversidade, São Paulo, v. 1, n. 1, p. 147155, jul. 2005.

MATHERON, G. Principles of geostatistics. Economic Geology, Lancaster, v. 58, p. 1246-1266, 1963.

MCBRATNEY, A. G.; WEBSTER, A. G. Choosing functions for semi-variograms and fitting them to sampling estimates. Journal of Soil Science, Oxford, v. 37, p. 617-639, 1986.

MCROBERTS, R. E.; NELSON, M. D.; WENDT, D. G. Stratified estimation of forest area using satellite imagery, inventory data, and the k-nearest neighbors technique. Remote Sensing of Environment, New York, v. 82, p. 457468, 2002.

MELLO, J. M.; BATISTA, J. L. F.; OLIVEIRA, M. F.; RIBEIRO JÚNIOR, P. J. Estudo da dependência espacial de características dendrométricas para Eucalyptus grandis. Cerne, Lavras, v. 11, n. 2, p. 113-126, 2005a.

MELLO, J. M.; BATISTA, J. L. F.; RIBEIRO JÚNIOR, P. J.; OLIVEIRA, M. S. Ajuste e seleção de modelos espaciais de semivariograma visando à estimativa volumétrica de Eucalyptus grandis. Scientia Forestalis, Piracicaba, n. 69, p. 25-37, dez. 2005b. 
MELLO, J. M.; DINIZ, F. S.; OLIVEIRA, A. D.; SCOLFORO, J. R. S.; ACERBI JÚNIOR, F. W.; THIERSCH, C. R. Métodos de amostragem geoestatística para estimativa do número de fustes e volume em plantios de Eucalyptus grandis. Floresta, Curitiba, v. 39, n. 1, p. 157-166, jan./mar. 2009.

MELLO, J. M.; OLIVEIRA, M. S.; BATISTA, J. L. F.; RIBEIRO JÚNIOR, P. J.; KANEGAE JÚNIOR, H. Uso do estimador geoestatístico para predição volumétrica por talhão. Floresta, Curitiba, v. 36, n. 2, maio/ago. 2006.

MELLO, J. M.; SCOLFORO, J. R. S. Análise comparativa de procedimentos de amostragem em um remanescente de floresta estacional semidecídua montana. Revista Árvore, Viçosa, v. 24, n. 1, p. 55-62, jan./fev. 2000.

NIMER, E. Climatologia do Brasil. Rio de Janeiro: IBGE, 1979. $422 \mathrm{p}$.

PÉLLICO NETTO, S.; BRENA, D. A. Inventário florestal. Curitiba: [s.n.], 1997. 316 p.

RIBEIRO JÚNIOR, P. J. Métodos geoestatísticos no estudo da variabilidade espacial de parâmetros do solo. 1995. 99 f. Dissertação (Mestrado em Engenharia Florestal) - Escola Superior de Agricultura "Luiz de Queiroz", Piracicaba, 1995.
RUFINI, A. L.; SCOLFORO, J. R. S.; OLIVEIRA, A. D.; MELLO, J. M. Equações volumétricas para o cerrado sensu stricto em Minas Gerais. Cerne, Lavras, v. 16, n. 1, p. 1-11, jan./mar. 2010.

SCOLFORO, J. R. S. Manejo florestal. Lavras: UFLA/ FAEPE, 1998. 443 p.

SCOLFORO, J. R. S.; MELLO, J. M. de. Inventário florestal. Lavras: UFLA/FAEPE, 2006. 561 p.

SCOLFORO, J. R. S.; MELLO, J. M. de; OLIVEIRA, A. D. Inventário florestal de Minas Gerais: cerrado, florística, diversidade, similaridade, distribuição diamétrica e de altura, volumetria, tendências de crescimento e áreas aptas para manejo florestal. Lavras: UFLA, 2008. 216 p.

SILVA, S. T. da. Estratificação da fitofisionomia cerrado para inventário florestal utilizando geotecnologia. 2009. 68 p. Dissertação (Mestrado em Engenharia Florestal) - Universidade Federal de Lavras, Lavras, 2009.

VENDRUSCULO, L. G. Uso de índices de desempenho e do critério de Akaike para ajuste de modelos de semivariograma. Campinas: EMBRAPA, 2003. 5 p. (Comunicado Técnico, 58). 